\title{
FARADAY ROTATION MEASUREMENT WITH THE SMAP RADIOMETER
}

\author{
D. M. Le Vine Ind S. Abraham $^{2}$ \\ 1. Goddard Space Flight Center, Greenbelt, MD 20771 \\ 2. RS Information Systems, Goddard Space Flight Center, Greenbelt, MD 20771
}

\begin{abstract}
Faraday rotation is an issue that needs to be taken into account in remote sensing of parameters such as soil moisture and ocean salinity at L-band. This is especially important for SMAP because Faraday rotation varies with azimuth around the conical scan. SMAP retrieves Faraday rotation in situ using the ratio of the third and second Stokes parameters, a procedure that was demonstrated successfully by Aquarius. This manuscript reports the performance of this algorithm on SMAP. Over ocean the process works reasonably well and results compare favorably with expected values. But over land, the inhomogeneous nature of the scene results in much noisier, and in some case unreliable, estimates of Faraday rotation.
\end{abstract}

\section{Index Terms-Microwave Remote Sensing, Faraday rotation, L-band}

\section{INTRODUCTION}

Faraday rotation is an issue that needs to be considered in the retrieval of ocean salinity and soil moisture at L-band $(1.4 \mathrm{GHz})$ where these measurements are made. As a result, each of the recent L-band missions in space, SMOS, Aquarius and SMAP, have included measurements to provide an in-situ estimates of Faraday rotation. In principle, Faraday rotation can be obtained from the ratio of the third and second Stokes parameter [8], and this was demonstrated successfully over oceans by Aquarius [6]. The same procedure is implemented in SMAP. However, the SMAP geometry (conical scanning) and the need to operate over both ocean (for retrieval of salinity) and over land (for retrieval of soil moisture) complicates the retrieval. The conical scan results in a rapid change of the viewing angle with respect to the local Earth magnetic field (with associated changes in Faraday rotation), and issues such as vegetation canopy and inhomogeneity of the scene result in noisy and sometimes unreliable retrievals.

In this manuscript comparison is presented of the rotation angle retrieved from the SMAP radiometer with expected values to illustrate the issues over land. The expected value is obtained using the TEC (total electron content) from the
International GNSS Service [4] and using the local value of the Earth magnetic field [2]. Research is also underway to compare the results over ocean with independent in situ measurements obtained over the ocean using retrievals from instruments on the Jason-2 spacecraft [1] at points of colocation as has been done for Aquarius [6].

In general, the global patterns and mean value along track agree reasonably well with the predictions. Over the ocean, the values of retrieved angle at individual points around the scan also compare reasonably well. However, over land, the retrieval is noisy and can be unstable. Over dense vegetation the retrieval can become unstable in the presence of noise and large errors are often observed over scenes that include large changes in the brightness temperature such as land-water boundaries.

\section{PROCEDURE}

As an initial step, a theoretical analysis has been conducted of the use of the ratio of the third and second Stokes parameter [8] to retrieve Faraday rotation in the SMAP geometry [7]. This uses a forward algorithm [5] that propagates signals from the surface to the radiometer to predict the two Stokes parameters that has been adapted to the SMAP geometry (conical scan and antenna patterns). The algorithm is similar to the SMAP simulator [3] and uses the same ancillary data for the surface (e.g. vegetation canopy and soil moisture) whenever possible. It uses the local Earth magnetic field [2] and total electron content from IGS to account for Faraday rotation. The purpose of the theoretical analysis is to understand a priori the issues associated with a conical scan and operating over land and to help understand features of the actual retrievals by SMAP. Then, a comparison was made of the Faraday rotation retrieved using the SMAP measurements of the second and third Stokes parameters with predictions based on the TEC reported by the IGS [4]. As part of this retrieval, a correction is applied to observed antenna temperature to correct for cross-polarization coupling in the antenna that improves the retrieval [7]. Research is also underway to compare the retrievals with independent in situ measurements obtained by co-located instruments on the Jason-2 spacecraft as done in the past for Aquarius [6]. 


\section{EXAMPLES}

Figures 1a-c show a comparison of the Faraday rotation angle retrieved by SMAP with the prediction based on the IGS TEC. Figure 1a (top) shows the SMAP orbit descending (right) and ascending (left) with the Faraday rotation angle (color coded) retrieved looking in the forward direction along the ground track (zero scan azimuth). Figure

(a)

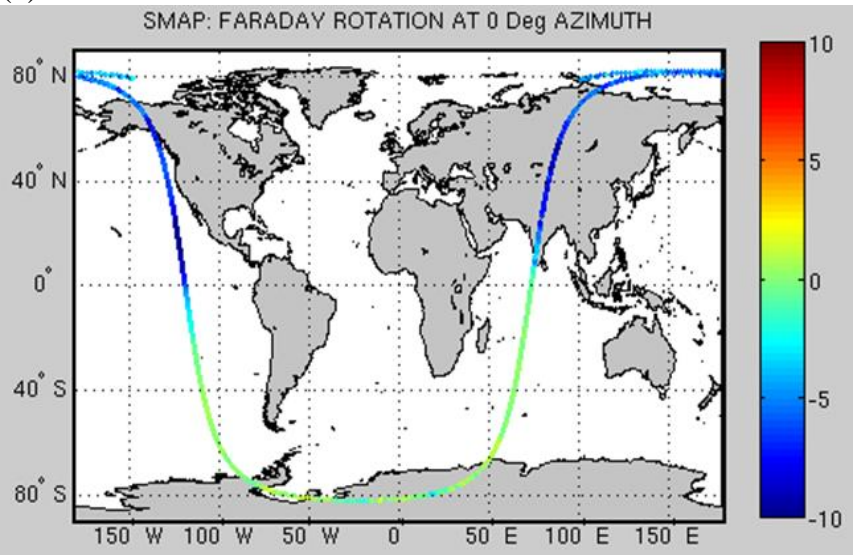

(b)

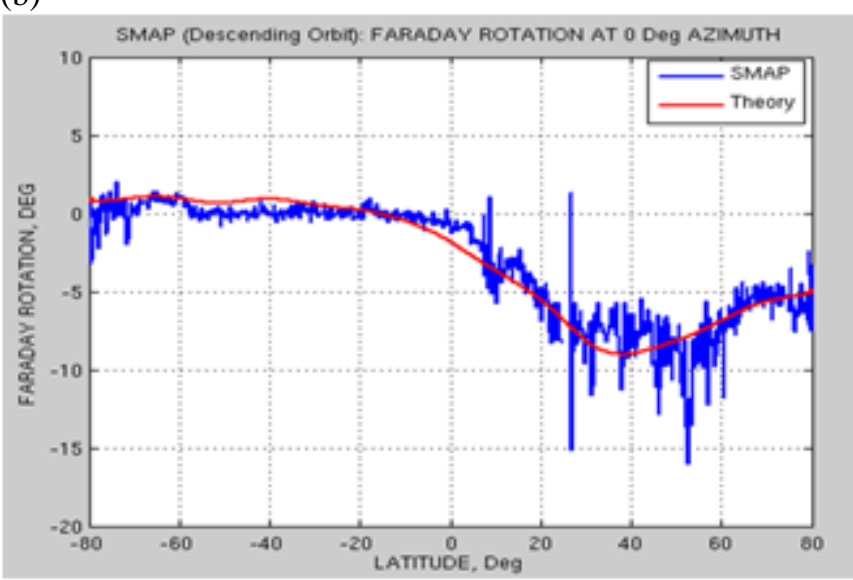

(c)

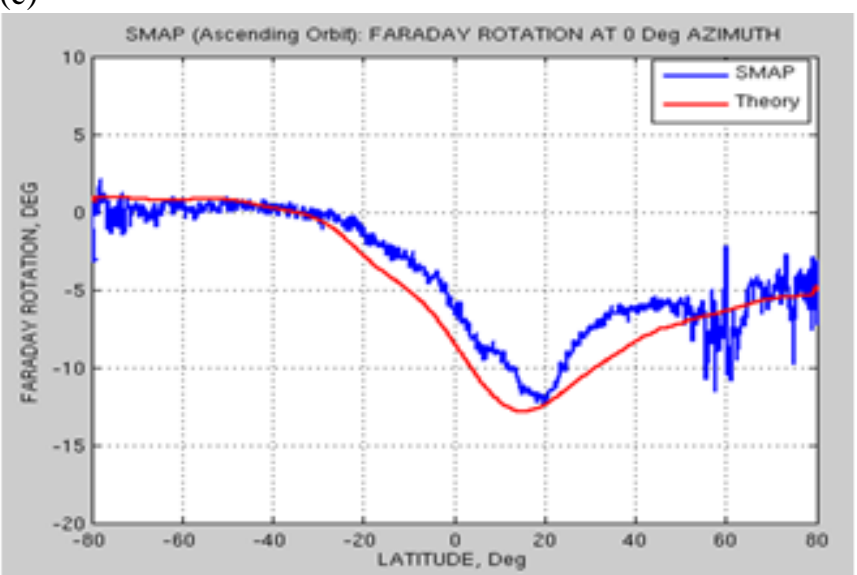

Figure 1: Comparison of predicted (model) and measured Faraday rotation angle.
$1 \mathrm{~b}$ shows the comparison of the retrieved angle (blue) and predicted angle using the IGS TEC (red; labelled "theory" in the key) along the descending portion of the orbit. (In Figure $1 \mathrm{~b}$, north is on the right and the satellite moves from right to left.) In general there is good agreement of measurement and prediction but the retrieved angle is much noisier over land (positive latitude). The noisier results over land correlate well with inhomogeneity of the scene and dense vegetation canopy and are predicted by theory [7]. Similar results can be seen in the ascending orbit (Figure 1c; bottom) but in this case the agreement over ocean is poorer in the vicinity of the peak in rotation angle near $20^{\circ}$ North latitude. Although not yet proven, there is evidence to suggest that this is due to poor estimate of the IGS TEC in the vicinity of these peaks.

This difference in behavior (i.e. good agreement over ocean, a relatively homogeneous scene) and noisy behavior over land is also evident in global maps of the retrieved Faraday rotation angle. This is illustrated in Figure 2
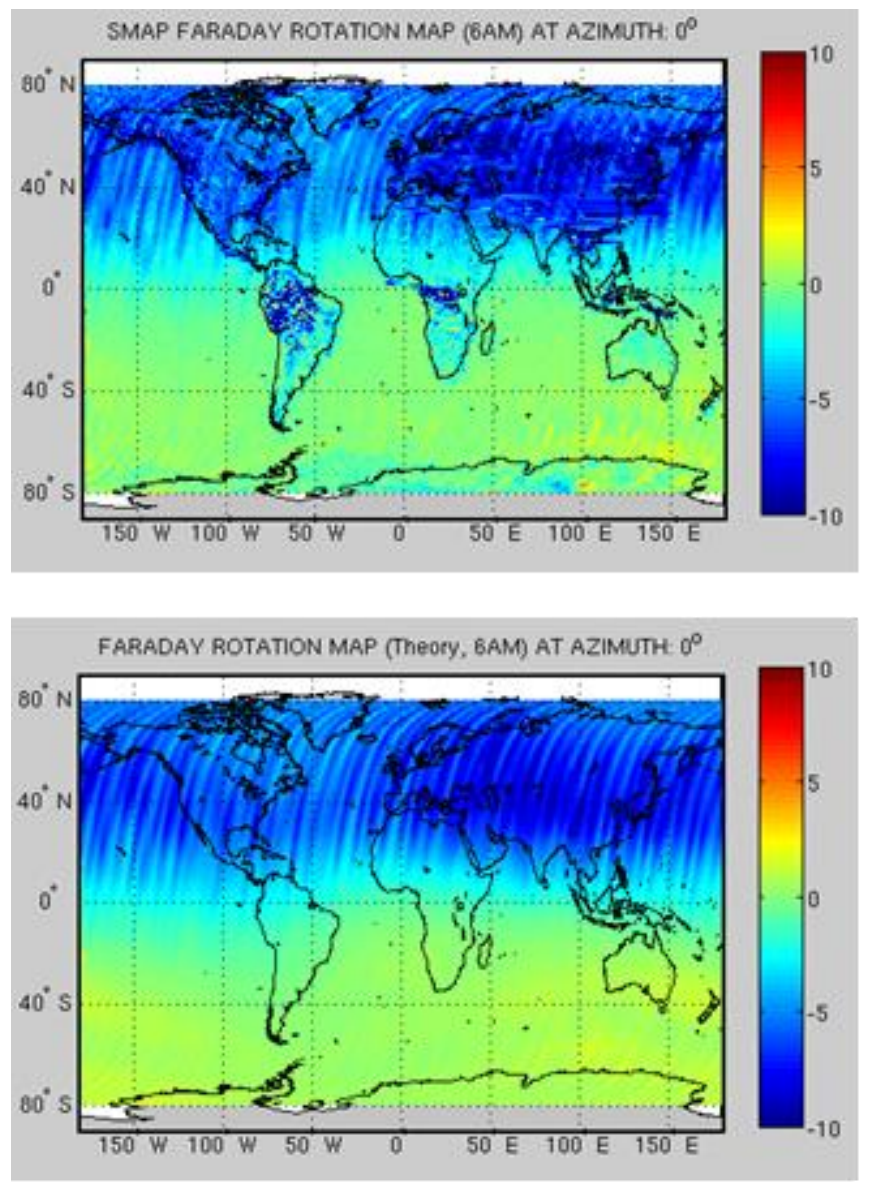

Figure 2: Global maps of Faraday rotation. (Top) Retrieved from SMAP measurements; (Bottom) Prediction using the TEC from IGS. 
Figure 2 shows the Faraday rotation angle in the forward direction (i.e. zero scan angle as in Figure 1) but with complete coverage of the globe. The top panel is the retrieved value (value obtained from SMAP measurements) and the bottom panel is the prediction using IGS (labelled "theory" in the figure). Notice in particular that there is no difference in the predicted values (bottom panel) over land or ocean; however, there are significant differences (the dark blue blotches) over land especially in areas of significant vegetation such as in South America in Brazil and south of the Sahara in Africa. They are also evident along the Northwest coast of North America.

The problems that occur over land can be seen more clearly when looking at the differences between retrieved and predicted (i.e. theoretical) values. This is shown in Figure 3 which shows the difference of the value obtained using the IGS TEC and the value retrieved by SMAP. There is a clear association of the larger differences with areas of dense vegetation and inhomogeneity in the scene (i.e. areas with lakes and mountains). The comparison in this case (Figure 3 ) is for a fixed scan angle of 120 degrees.

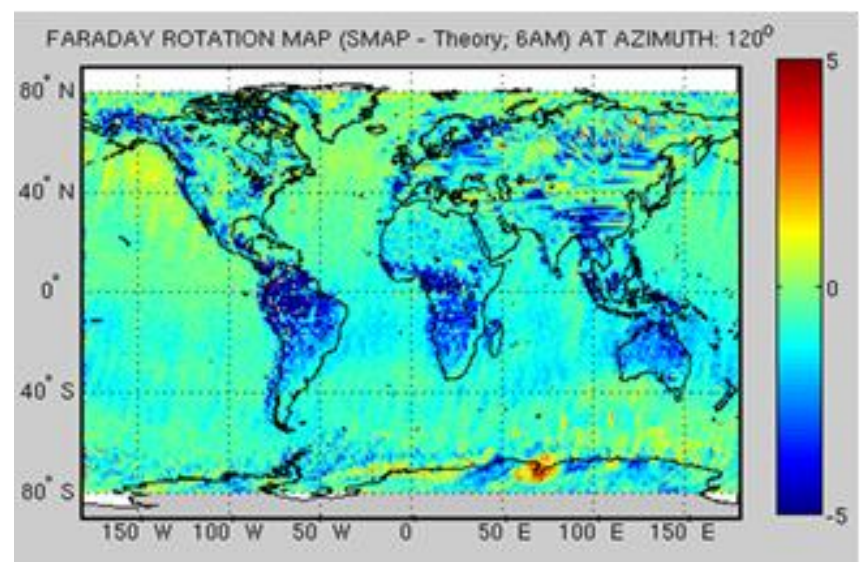

Figure 3: Difference between retrieved and predicted Faraday rotation angle at a scan angle of 120 degrees.

\section{CONCLUSIONS}

Examples are presented here of the Faraday rotation angle retrieved from the third and second Stokes parameters measured by SMAP using the algorithm proposed by Yueh [8] and validated by Aquarius [6]. Over the ocean, a relatively homogeneous scene, the retrieved values are in reasonably good agreement with values predicted using TEC from the IGS. The behavior over land is not as good and there are large differences which can be associated with inhomogeneity of the scene and areas of dense vegetation. This behavior over land is consistent with predictions obtained from simulations (theory) using radiative transport models [5] [7] and has also been seen in the Aquarius measurements [6]. The retrieval algorithm has been validated over ocean using measurements from Aquarius with in situ measurements from instruments on the Jason-2 spacecraft [6] and work is underway to repeat this validation for SMAP.

\section{REFERENCES}

[1] W. J. Bannoura, A. Wade, and D. N. Srinivas, "NOAA ocean surface topography mission Jason-2 project overview," in Proc. MTS/IEEE OCEANS, 2005, vol. 3, pp. 2155-2159.

[2] C.E. Barton, "International Geomagnetic Reference Field: The Seventh Generation", J. Geomag. Geolectr., Vol 49, pp 123-148, 1997.

[3] D. Entekhabi et al., "SMAP Handbook: Soil Moisture Active Passive,"Nat. Aeronaut. Space Admin., Jet Propulsion Lab., Pasadena, CA, USA, Jul. 2014.

[4] IGS 2015: http://www.igs.org

[5] D.M. Le Vine, E.P. Dinnat, S. Abraham, P. de Matthaeis, F. Wentz, "The Aquarius Simulator and Cold-Sky Calibration", IEEE Trans. Geoscience and Remote Sensing, Vol. 40 (\#9), pp 31983210, September 2011.

[6] D.M. Le Vine, S. Abraham, C. Utku and E.P. Dinnat, "Aquarius Third Stokes Parameter Measurements: Initial Results", IEEE Geoscience and Remote Sensing Letters, Vol.10, no. 3, pp 520-524, May 2013.

[7] D.M. Le Vine, S. Abraham, J. Peng, "Faraday Rotation Correction for the SMAP Radiometer", IEEE Trans. Geoscience and Remote Sensing, Vol. 54 (\#4), pp 2070-2081, 2016.

[8] S.Yueh, "Estimates of Faraday Rotation with Passive Microwave Polarimetry for Microwave Remote Sensing of Earth Surfaces", IEEE Trans. Geoscience and Remote Sensing, Vol. 38 (\# 5), pp 2434-2438, 2000. 